Supplementary Information

\title{
Kinetic Investigation on the Cationic Polymerization of o-Phthalaldehyde: Understanding Ring-Expansion Polymerization
}

\author{
Anthony Engler, Paul A. Kohl*
}

School of Chemical and Biomolecular Engineering, Georgia Institute of Technology, Atlanta, Georgia 30332, United States.

*Corresponding author: kohl@gatech.edu

1. General Experimental Details

S2

2. Polymerization Data

S4 


\section{General Experimental Details}
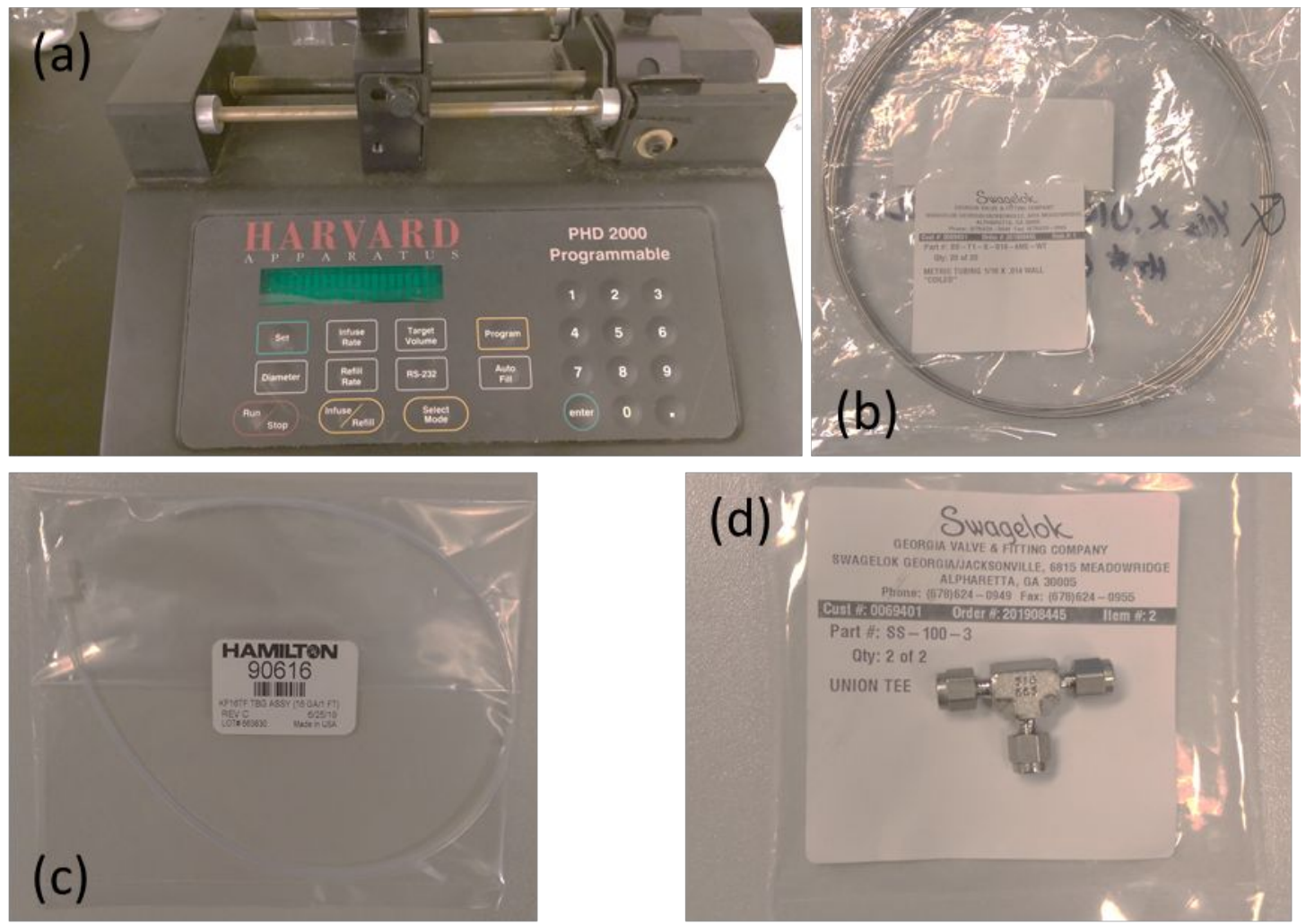

Figure S1. Pictures of flow reactor parts. (a) syringe pump; (b) 316 stainless steel pipe, 1/16" OD x 0.014 in wall thickness; (c) PTFE luer-lock adapter and tubing, 12" long, 1/16" ID; (d) 316 stainless steel union tee for $1 / 16$ " OD pipe.

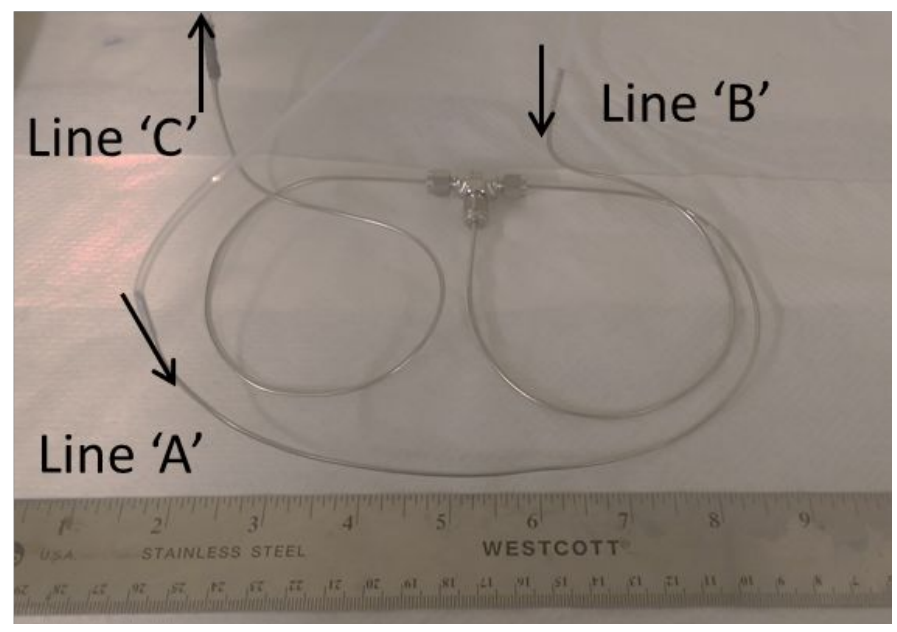


Figure S2. Picture of constructed stainless steel reactor portion.

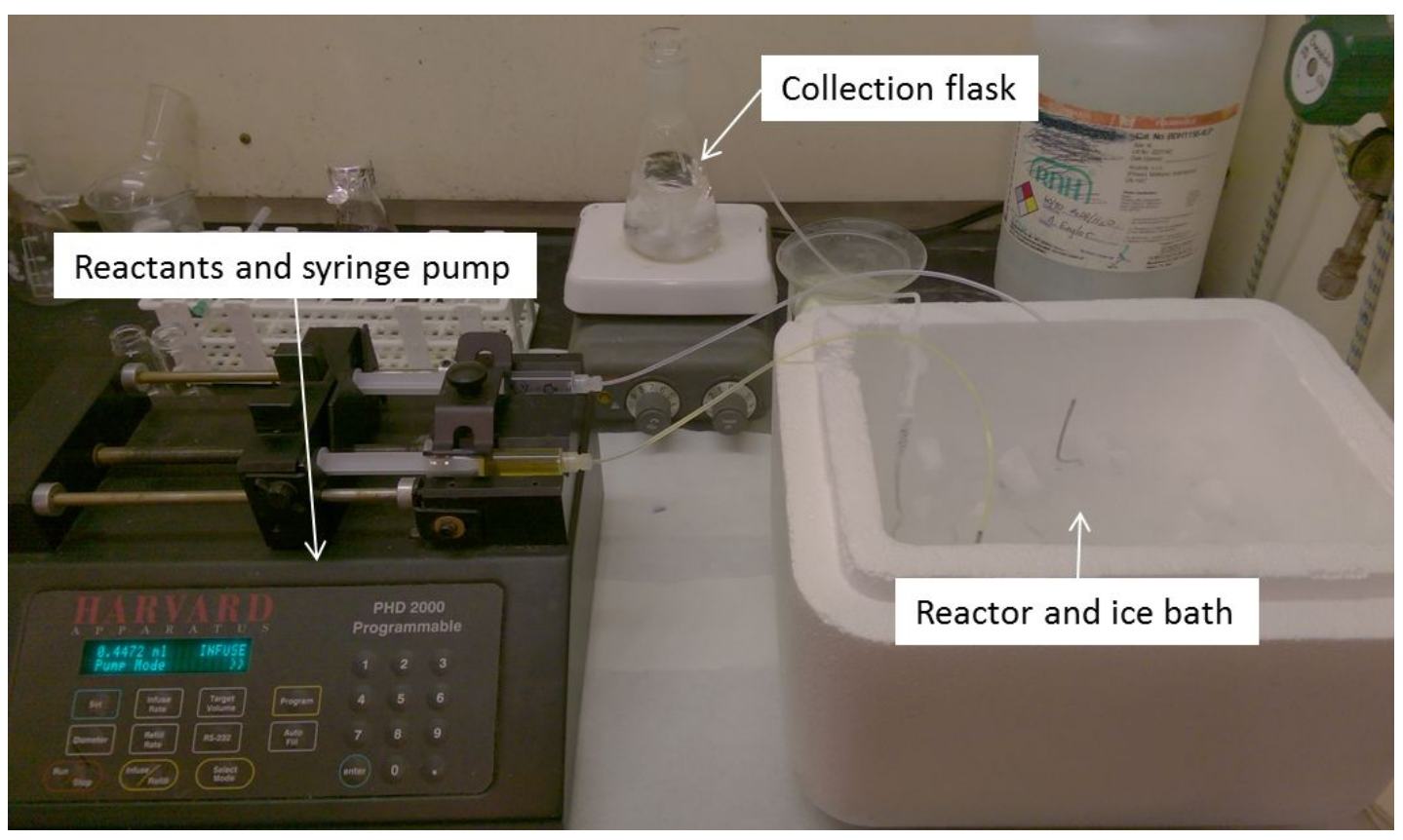

Figure S3. Flow reactor set up during operation.

Table S1. Flow reactor parameters. See Figure S2 for identification of segment lines.

\begin{tabular}{|l|l|l|l|}
\hline Length of segment 'A' & 12 in & Volume & $187 \mu \mathrm{L}$ \\
\hline Length of segment ' $B$ ' & 10 in & & \\
\hline Length of segment 'C' & 10 in & & \\
\hline Internal diameter of steel pipes & $876 \mu \mathrm{m}$ & & \\
\hline Surface area-to-volume ratio of segment ' $\mathrm{A}$ ' & $4571 \mathrm{~m}^{-1}$ & & \\
\hline
\end{tabular}




\section{Polymerization data}

Flow Polymerizations

Table S2. Polymerization data for the flow reactor experiments with $[\mathrm{M}] /[\mathrm{I}]=500$ and $\mathrm{T}=-78^{\circ} \mathrm{C}$.

\begin{tabular}{|l|l|l|l|}
\hline \multicolumn{1}{|c|}{$\boldsymbol{\tau}_{\mathbf{R}}(\mathbf{s})$} & \multicolumn{1}{c|}{ Yield (\%) } & \multicolumn{1}{c|}{$\mathbf{M}_{\mathbf{n}}(\mathbf{k D a})$} & $\boldsymbol{D}$ \\
\hline 2.00 & 4.3 & 40.8 & 2.02 \\
\hline 4.00 & 24.3 & 68.7 & 1.96 \\
\hline 7.00 & 30.9 & 73.6 & 1.88 \\
\hline 10.00 & 34.3 & 77.4 & 1.67 \\
\hline 20.04 & 47.8 & 80.9 & 2.03 \\
\hline 29.93 & 66.6 & 89 & 1.85 \\
\hline 44.93 & 72.5 & 129 & 2.04 \\
\hline 59.93 & 80.7 & 133 & 2.08 \\
\hline 90.00 & 85.8 & 137 & 1.84 \\
\hline 121.78 & 89.6 & 188 & 1.94 \\
\hline
\end{tabular}

Table S3. Polymerization data for the flow reactor experiments with $[\mathrm{M}] /[\mathrm{I}]=300$ and $\mathrm{T}=-78^{\circ} \mathrm{C}$.

\begin{tabular}{|l|l|l|l|}
\hline \multicolumn{1}{|c|}{$\boldsymbol{\tau}_{\mathrm{R}}(\mathbf{s})$} & \multicolumn{1}{c|}{ Yield (\%) } & \multicolumn{1}{c|}{$\mathbf{M}_{\mathbf{n}}(\mathbf{k D a})$} & $\boldsymbol{D}$ \\
\hline 2.00 & 23.7 & 34.2 & 2.47 \\
\hline 4.00 & 40.4 & 50.5 & 2.32 \\
\hline 7.00 & 51.5 & 66.2 & 2.01 \\
\hline 10.00 & 57.1 & 63.6 & 1.81 \\
\hline 20.04 & 70.5 & 64.8 & 1.74 \\
\hline 29.93 & 77.6 & 77.8 & 2.29 \\
\hline 44.93 & 82.5 & 81.7 & 2.16 \\
\hline 59.93 & 85.8 & 99.2 & 2.10 \\
\hline 80.34 & 88.9 & 101 & 1.81 \\
\hline 100.05 & 92.2 & 137 & 1.87 \\
\hline
\end{tabular}

Table S4. Polymerization data for the flow reactor experiments with $[\mathrm{M}] /[\mathrm{I}]=160$ and $\mathrm{T}=-78^{\circ} \mathrm{C}$.

\begin{tabular}{|l|l|l|l|}
\hline \multicolumn{1}{|c|}{$\boldsymbol{\tau}_{\mathbf{R}}(\mathbf{s})$} & \multicolumn{1}{c|}{ Yield (\%) } & \multicolumn{1}{c|}{$\mathbf{M}_{\mathbf{n}}(\mathbf{k D a})$} & $\boldsymbol{\Xi}$ \\
\hline 1.00 & 8.97 & 66.7 & 2.24 \\
\hline 2.00 & 45.5 & 126 & 2.10 \\
\hline 4.00 & 56.1 & 139 & 2.07 \\
\hline 7.00 & 78.9 & 170 & 2.08 \\
\hline 10.00 & 85.9 & 198 & 2.01 \\
\hline
\end{tabular}




\begin{tabular}{|l|l|l|l|}
\hline 20.04 & 90.4 & 244 & 2.06 \\
\hline 29.93 & 92.3 & 252 & 2.26 \\
\hline 44.93 & 94.3 & 276 & 1.94 \\
\hline
\end{tabular}

Table S5. Polymerization data for the flow reactor experiments with $[\mathrm{M}] /[\mathrm{I}]=50$ and $\mathrm{T}=-78^{\circ} \mathrm{C}$.

\begin{tabular}{|l|l|l|l|}
\hline \multicolumn{1}{|c|}{$\boldsymbol{\tau}_{\mathbf{R}}(\mathbf{s})$} & \multicolumn{1}{c|}{ Yield (\%) } & \multicolumn{1}{c|}{$\mathbf{M}_{\mathbf{n}}(\mathbf{k D a})$} & \multicolumn{1}{c|}{$\boldsymbol{\oplus}$} \\
\hline 0.50 & 6.67 & 91.6 & 2.22 \\
\hline 1.00 & 45.3 & 130 & 2.11 \\
\hline 2.00 & 65.6 & 140 & 2.33 \\
\hline 4.00 & 85.6 & 144 & 2.37 \\
\hline 7.00 & 91.4 & 177 & 2.14 \\
\hline 10.00 & 94.4 & 277 & 2.13 \\
\hline
\end{tabular}

Table S6. Polymerization data for the flow reactor experiments with $[\mathrm{M}] /[\mathrm{l}]=160$ and $\mathrm{T}=-57^{\circ} \mathrm{C}$. Sample with $\tau_{R}=1.00$ did not have enough material for a GPC sample. Data points below the thick horizontal line are beyond the linear portion of the reaction and highlight the pinching reaction affects.

\begin{tabular}{|l|l|l|l|}
\hline \multicolumn{1}{|c|}{$\boldsymbol{\tau}_{\mathbf{R}}(\mathbf{s})$} & \multicolumn{1}{c|}{ Yield (\%) } & \multicolumn{1}{c|}{$\mathbf{M}_{\mathbf{n}}(\mathbf{k D a})$} & \multicolumn{1}{c|}{$\boldsymbol{D}$} \\
\hline 1.00 & 0.28 & - & - \\
\hline 2.00 & 30.3 & 87 & 2.14 \\
\hline 4.00 & 48.3 & 106 & 2.03 \\
\hline 7.00 & 55.9 & 120 & 2.15 \\
\hline 10.00 & 56.9 & 85.8 & 2.08 \\
\hline 20.00 & 55.5 & 68.5 & 1.95 \\
\hline
\end{tabular}

Table S7. Polymerization data for the flow reactor experiments with $[\mathrm{M}] /[\mathrm{l}]=160$ and $\mathrm{T}=-67^{\circ} \mathrm{C}$.

\begin{tabular}{|l|l|l|l|}
\hline \multicolumn{1}{|c|}{$\boldsymbol{\tau}_{\mathbf{R}}(\mathbf{s})$} & \multicolumn{1}{c|}{ Yield (\%) } & \multicolumn{1}{c|}{$\mathbf{M}_{\mathbf{n}}(\mathbf{k D a})$} & \multicolumn{1}{c|}{$\boldsymbol{D}$} \\
\hline 1.00 & 2.91 & 40 & 2.87 \\
\hline 2.00 & 37.3 & 122 & 2.14 \\
\hline 4.00 & 62.4 & 154 & 2.20 \\
\hline 7.00 & 70.3 & 155 & 2.17 \\
\hline 10.00 & 78.7 & 174 & 2.09 \\
\hline
\end{tabular}




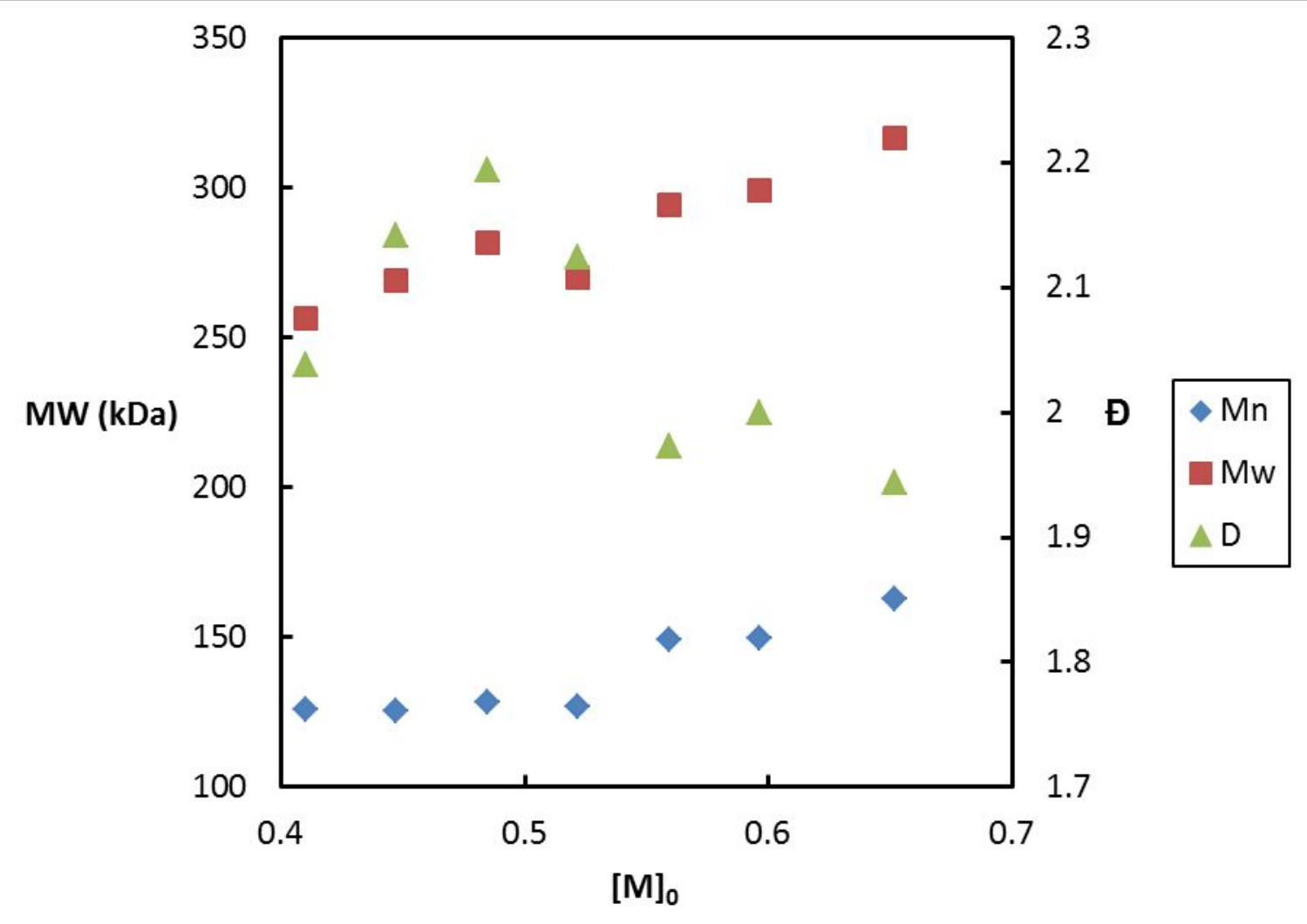

Figure S4. Equilibrium batch polymerization data systematically varying the $[M]_{0}$ values to show that large polymer is formed before this kinetics transition, and therefore likely not due to cyclic critical concentrations as given by Jacobson-Stockmayer theory. 